\title{
Práticas de saúde oral em idosos com demência: Revisão sistemática
}

\author{
Oral health practices for elderly patients with dementia: A systematic review \\ Prácticas de salud oral en personas mayores con demencia: Una revisión sistemática
}

Recebido: 18/07/2021 | Revisado: 25/07/2021 | Aceito: 27/07/2021 | Publicado: 03/08/2021

\author{
Érica Paula Barbosa \\ ORCID: https://orcid.org/0000-0002-8280-4699 \\ Universidade Estadual de Ciências da Saúde de Alagoas, Brasil \\ E-mail: erica.barbosa@academico.uncisal.edu.br \\ Paulla Valéria de Souza Meneses \\ ORCID: https://orcid.org/0000-0001-6749-9377 \\ Universidade Estadual de Ciências da Saúde de Alagoas, Brasil \\ E-mail: paullavaleria@hotmail.com \\ Cinthya Rafaella Magalhães da Nóbrega Novaes \\ ORCID: https://orcid.org/0000-0003-4795-6328 \\ Universidade Estadual de Ciências da Saúde de Alagoas, Brasil \\ E-mail: rafamn20@yahoo.com.br \\ Ana Renata Lima Leandro \\ ORCID: https://orcid.org/0000-0003-2707-0730 \\ Universidade Estadual de Ciências da Saúde de Alagoas, Brasil \\ E-mail: releandro@gmail.com \\ Vivianne de Lima Biana Assis \\ ORCID: https://orcid.org/0000-0002-5110-7698 \\ Universidade Estadual de Ciências da Saúde de Alagoas, Brasil \\ E-mail: vivibiana@gmail.com \\ Monique Carla da Silva Reis \\ ORCID: https://orcid.org/0000-0002-8815-3938 \\ Universidade Estadual de Ciências da Saúde de Alagoas, Brasil \\ E-mail: moniquecsto@gmail.com
}

\begin{abstract}
Resumo
O presente estudo objetivou analisar as práticas odontológicas em pacientes idosos com demência através de uma revisão sistemática. por meio de pesquisa eletrônica nas bases de dados PubMed, Scientific Electronic Library Online (Scielo), Web of Science, Virtual Health Library ou Biblioteca Virtual em Saúde (BVS), Scopus, Google Acadêmico, nos meses de setembro a novembro de 2020. A literatura cinzenta foi incluída através da Biblioteca Digital de Teses e Dissertações e da OpenThesis. A busca utilizou a estratégia PECOS (P: paciente; E; exposição; Comparação; O desfecho; S tipo de estudo) através da pergunta de pesquisa: Como são conduzidas as práticas odontológicas em pacientes idosos com demência? Foram considerados elegíveis estudos observacionais (transversal, caso-controle e coorte), nos quais os participantes da pesquisa possuíam diagnóstico de algum tipo de demência, com idade igual ou superior a 60 anos, e que essas práticas fossem realizadas na assistência primária ou domiciliar. Os artigos incluídos foram selecionados por dois revisores de forma independente e os desacordos resolvidos por um terceiro revisor. A avaliação da qualidade metodológica dos estudos selecionados foi realizada por meio da Escala de Newcastle-Ottawa, que avalia o risco de viés. A pesquisa apontou que três artigos selecionados relatam a inserção de práticas odontológicas a pacientes idosos com demência, sendo dois de qualidade moderada e um de baixa qualidade. Deste modo, conclui-se que os resultados obtidos mostram a necessidade de estudos com delineamentos mais rigorosos, com menor risco de viés, como os ensaios clínicos controlados e randomizados, com o objetivo de testar hipóteses e produzir protocolos eficientes para o desenvolvimento de técnicas para as intervenções odontológicas aos idosos com demência.
\end{abstract}

Palavras-chave: Demência; Idosos; Saúde Bucal.

\begin{abstract}
The present study aimed to analyze the dental practices in elderly patients with dementia through a systematic review. through electronic search in the databases PubMed, Scientific Electronic Library Online (Scielo), Web of Science, Virtual Health Library or Virtual Health Library (VHL), Scopus, Google Academic, in the months of September to November 2020. Gray literature was included through the Digital Library of Theses and Dissertations and OpenThesis. The search used the PECOS strategy (P: patient; E; exposure; Comparison; O outcome; S type of study) through the research question: How are dental practices conducted in elderly patients with dementia? Observational studies (crosssectional, case-control, and cohort) were considered eligible, in which the research participants had a diagnosis of some type of dementia, aged 60 years or older, and that these practices were conducted in primary care or at home. The included articles were independently selected by two reviewers and disagreements were resolved by a third reviewer.
\end{abstract}


The methodological quality of the selected studies was assessed using the Newcastle-Ottawa Scale, which evaluates the risk of bias. The research pointed out that three selected articles report the insertion of dental practices to elderly patients with dementia, two of moderate quality and one of low quality. Thus, we conclude that the results obtained show the need for studies with more rigorous designs, with lower risk of bias, such as controlled and randomized clinical trials, in order to test hypotheses and produce efficient protocols for the development of techniques for dental interventions for the elderly with dementia.

Keywords: Dementia; Elderly; Oral health.

\section{Resumen}

El presente estudio tuvo como objetivo analizar las prácticas odontológicas en pacientes ancianos con demencia a través de una revisión sistemática. mediante la búsqueda electrónica en las bases de datos PubMed, Scientific Electronic Library Online (Scielo), Web of Science, Virtual Health Library o Biblioteca Virtual de Salud (BVS), Scopus, Google Academic, en los meses de septiembre a noviembre de 2020. La literatura gris se incluyó a través de la Biblioteca Digital de Tesis y Disertaciones y OpenThesis. La búsqueda utilizó la estrategia PECOS (P: paciente; E; exposición; Comparación; O resultado; S tipo de estudio) a través de la pregunta de investigación: ¿Cómo se realizan las prácticas odontológicas en los pacientes ancianos con demencia? Se consideraron elegibles los estudios observacionales (transversales, de casos y controles y de cohortes) en los que los participantes en la investigación tuvieran un diagnóstico de algún tipo de demencia, tuvieran 60 años o más, y que estas prácticas se realizaran en atención primaria o en el domicilio. Los artículos incluidos fueron seleccionados independientemente por dos revisores y los desacuerdos fueron resueltos por un tercer revisor. La evaluación de la calidad metodológica de los estudios seleccionados se realizó mediante la escala Newcastle-Ottawa, que evalúa el riesgo de sesgo. La investigación señaló que tres artículos seleccionados informan sobre la inserción de prácticas dentales a pacientes ancianos con demencia, dos de calidad moderada y uno de calidad baja. Así, se concluye que los resultados obtenidos muestran la necesidad de realizar estudios con diseños más rigurosos, con menor riesgo de sesgo, como los ensayos clínicos controlados y aleatorizados, para poder comprobar las hipótesis y elaborar protocolos eficientes para el desarrollo de técnicas de intervención odontológica en pacientes ancianos con demencia.

Palabras clave: Demencia; Ancianos; Salud bucal

\section{Introdução}

No Brasil, a Política Nacional do Idoso caracteriza como velho o indivíduo com mais de 60 anos, onde permear as fronteiras da velhice traz desacordo, mesmo a velhice sendo apontada como fenômeno biológico. Sem dúvida, a maioria dos idosos sofrem, em maior ou menor grau, de perda de agilidade e de mais doenças do que os jovens (Cardoso, Dietrich \& Souza, 2021). Nesse sentido, o envelhecimento no Brasil possui uma importante associação com os determinantes sociais em saúde, sendo a renda um dos determinantes mais relevantes (Duque, 2019).

Neste momento, as pessoas com 60 anos ou mais já retratam 12,1\% da população brasileira total (Instituto Brasileiro de Geografia e Estatística, 2013). Dados da Organização Mundial da Saúde (OMS) contabilizam que, em 2025, o Brasil será o sexto país no mundo com o maior número de idosos (Organização Mundial de Saúde, 2005). A expectativa de vida no país, atualmente, é de 75,7 anos e em 2030 e 2060 subirá para 78,7 e 81,2 anos respectivamente. A repercussão desse avanço, que em 2030 poderá exceder 40 milhões e, em 2060 ultrapassar 60 milhões de pessoas no Brasil, possui impactos na economia, no mercado de trabalho, nas relações familiares e no sistema de saúde (Instituto Brasileiro de Geografia e Estatística, 2013). O aumento da expectativa de vida, a redução das taxas de natalidade e a ampliação da assistência à saúde foram responsáveis pelo aumento da população idosa brasileira (Paim et al., 2011).

Nessa fase da vida, o idoso consome mais serviços de saúde, as internações são mais frequentes e o tempo de ocupação do leito é maior. Em geral, os idosos são acometidos por doenças crônicas e múltiplas. As doenças crônicas não transmissíveis (DCNT) e podem comprometer a capacidade funcional do idoso, sendo as principais causas de incapacidade e mortalidade em todo o mundo (Dantas, 2013). Em um levantamento realizado pela (Organização Mundial de Saúde, 2005), as principais DCNT e agravos que afetam o idoso foram: doenças cardiovasculares, hipertensão, acidente vascular cerebral, diabetes, neoplasias, doença pulmonar obstrutiva crônica, doenças musculoesqueléticas (como artrite e artrose), demência, cegueira e diminuição da visão, doenças mentais e depressão. 
A saúde bucal e o atendimento odontológico são frequentemente negligenciados pelos pacientes e seus cuidadores, tendo em vista que outros agravos como hipertensão, cardiopatias, são priorizados. Além disso, pacientes com demência geralmente sofrem de sintomas comportamentais e psiquiátricos, que podem levar a comportamentos de resistência a esses cuidados, resultando em dificuldades na realização da higiene bucal, ao passo que esse paciente deve ir regularmente ao dentista, afim de manter a higiene oral e reduzir a perda dentária, assim como presença de dor e outros agravos ocorridos em consequência da ausência do cuidado, bem como seus cuidadores serem orientados sobre a importância dessa higiene bucal (Fereshtehnejad et al., 2018; Hatipoglu, Kabay \& Guven, 2011; Zenthöfer et al., 2014).

Diante disso, foi realizada uma revisão sistemática para compreender as práticas odontológicas em pacientes idosos com demência.

\section{Metodologia}

Trata-se de uma revisão sistemática da literatura sobre as práticas odontológicas em pessoa com demência. As revisões sistemáticas são consideradas estudos secundários, que têm nos estudos primários sua fonte de dados, sendo um estudo rigoroso, uma escrita de averiguação com técnicas sistemáticas pré definidas para descrever sistematicamente os estudos pertinentes publicados e não publicados, onde para uma questão de análise, considera a qualidade desses artigos, extrai dados e sintetiza os resultados (Donato \& Donato,2019). O estudo seguiu as recomendações do método Preferred Reporting Items for Systematic Reviews and Meta-Analyses (PRISMA), e os autores adotaram os seguintes procedimentos: a) definição da problemática e proposta de revisão; b) elaboração do formulário de registro; c) definição das equações de pesquisa (descritores e combinações) e identificação das fontes; d) âmbito da pesquisa (tipo de material e período); d) critérios de inclusão e exclusão); identificação, avaliação da qualidade e seleção de estudo primário por juízes; f) extração de dados, análise e síntese dos resultados.

O protocolo desta revisão sistemática foi registrado no banco de dados do International Prospective Register of Systematic Reviews (PROSPERO) sob registro CRD42020214662, e apresentado de acordo com a lista de verificação de Principais Itens para Relatar Revisões Sistemáticas e Meta-análises.

As buscas foram realizadas nas bases de dados PubMed, Scientific Electronic Library Online (Scielo), Web of Science, Virtual Health Library ou Biblioteca Virtual em Saúde (VHL ou BVS), Scopus, Google Acadêmico, no período de setembro a novembro 2020. A literatura cinzenta foi incluída através da Biblioteca Digital de Teses e Dissertações e da OpenThesis. Além disso, foi realizada uma busca manual nos artigos selecionados através da lista de referências para identificar eventuais artigos que pudessem preencher os critérios de elegibilidade do estudo no caso de não haver encontrado na busca inicial. A estratégia de busca foi apresentada em português e inglês e uma para cada base. Não houve restrição em relação ao idioma ou ao ano de publicação dos estudos.

Para compor a estratégia de busca, foi utilizada a estratégia PECOS (Quadro 1), os termos de busca foram definidos a partir do Medical Subject Heading (MeSH) e do Descritores em Ciências da Saúde (DeCS). Para estruturar a estratégia de busca, foram combinados os seguintes termos MeSH: “dentist”, “oral heath”, “dental care”, “dementia” e "Alzheimer”, assim como os seus sinônimos e entre termos, e DeCS correspondentes. Não foram utilizados filtros durante a busca, para ampliar o número de artigos elegíveis. A partir da estratégia PECOS (Quadro 1), a seguinte pergunta de pesquisa foi definida: Como são conduzidas as práticas odontológicas em pacientes idosos com demência? 
Quadro 1 - Estratégia PECOS.

\begin{tabular}{|c|c|l|}
\hline Acrômio & Descrição do PECOS & \multicolumn{1}{|c|}{ Termos MeSH correspondentes } \\
\hline P & Idosos & Aged \\
Age Groups
\end{tabular}

Fonte: Autores.

No estudo foram incluídos artigos, teses e dissertações nos quais os participantes da pesquisa tinham diagnóstico de algum tipo de demência, com idade igual ou superior a 60 anos, e que essas práticas sejam realizadas na assistência primária, assistência domiciliar e Instituições de Longa Permanência (ILPs). Não puderam compor esta revisão os artigos que não condizem com a essência da pergunta da pesquisa, não disponíveis nas línguas inglês e/ou português, e aqueles em que não foi possível obter versão completa do artigo original, como as revisões da literatura e sistemáticas, relatórios e editoriais, além de estudos onde a intervenção não foi conduzida por dentistas.

Após a busca foi realizada a leitura de títulos e resumos por dois pesquisadores (E.P.B.) e (P.V.M.), de forma independente e cega. As discordâncias foram julgadas por um terceiro avaliador (M.C.S.R.) e em seguida, foi realizada a leitura dos artigos na íntegra, obedecendo rigorosamente aos critérios de inclusão e exclusão definidos para a pesquisa. A extração de dados foi realizada por dois investigadores (P.V.M.) e (E.P.B.), de forma independente e posteriormente comparadas. Os seguintes dados foram localizados, como: título do estudo, autor, ano de publicação, população, diagnóstico, local de intervenção, tipo de intervenção realizada, serviço de saúde, profissionais envolvidos na prática, tempo de avaliação, desfecho, revista de publicação e local do estudo.

A avaliação da qualidade metodológica dos estudos foi realizada por dois pesquisadores (E.P.B.) e (M.C.S.R.), onde foi utilizada a Escala de Newcastle-Ottawa (NOS) para estudos de coorte e a Escala de Newcastle-Ottawa modificada para estudos transversais (Terwee et al., 2007; Wells, 1999).

Essa adaptação da escala Newcastle-Ottawa para estudos transversais, se deu a partir da revisão sistemática, "Exposure to second-hand smoke and the risk of tuberculosis in children and adults: systematic review and a meta-analysis of 18 observational studies”, Patra et al. (2015), desde então, tem sido utilizada em vários estudos que possuem a necessidade de avaliar a qualidade deste tipo de estudo.

$\mathrm{Na}$ avaliação do risco de viés foram contemplados os seguintes domínios: seleção dos grupos (generalização e aplicabilidade), comparabilidade dos grupos no estudo, métodos para avaliação do desfecho (estudos de coorte). Cada item pode receber 1 ponto (uma estrela), exceto o item "Comparabilidade”, em que a pontuação varia de 0 a 2 estrelas. A pontuação máxima pode ser de 9 pontos para estudos de coorte e de 7 para estudos transversais, o que representa alta qualidade metodológica (Wells, 1999). 
Estudos de coorte de 6 a 8 estrelas foram classificados como moderados, e aqueles com 5 estrelas ou menos foram considerados de baixa qualidade. Estudos transversais com classificação entre 4 e 6 estrelas foram avaliados como moderado, e aqueles com 3 estrelas ou menos foram considerados de baixa qualidade.

O Fluxograma dos estudos incluídos foi formulado com base no cumprimento das etapas adotadas para a construção desta revisão, conforme os critérios de elegibilidade. Devido à ausência de heterogeneidade metodológica dos estudos e ausência de dados essenciais para realizar uma metanálise, optou-se por uma análise descritiva para substanciar os resultados dos estudos incluídos nesta revisão sistemática.

\section{Resultados}

Após a realização das buscas nas bases de dados, os autores obtiveram um total de 1.126 artigos identificados a partir da estratégia de busca adotada. Após exclusão dos títulos duplicados (13), foram selecionados 1.113 para a leitura dos títulos e resumos, restando sete artigos para a leitura na íntegra, onde seis desses trabalhos foram excluídos pelos critérios de elegibilidade.

Pesquisas manuais foram realizadas nas listas de referência dos artigos resgatados, com o objetivo de identificar estudos adicionais relevantes e não alcançados através da estratégia inserida nas bases de dados onde foram selecionados dois artigos, totalizando assim em três artigos para a inclusão nos resultados desta revisão sistemática. (Figura 1).

Figura 1- Fluxograma de seleção dos estudos

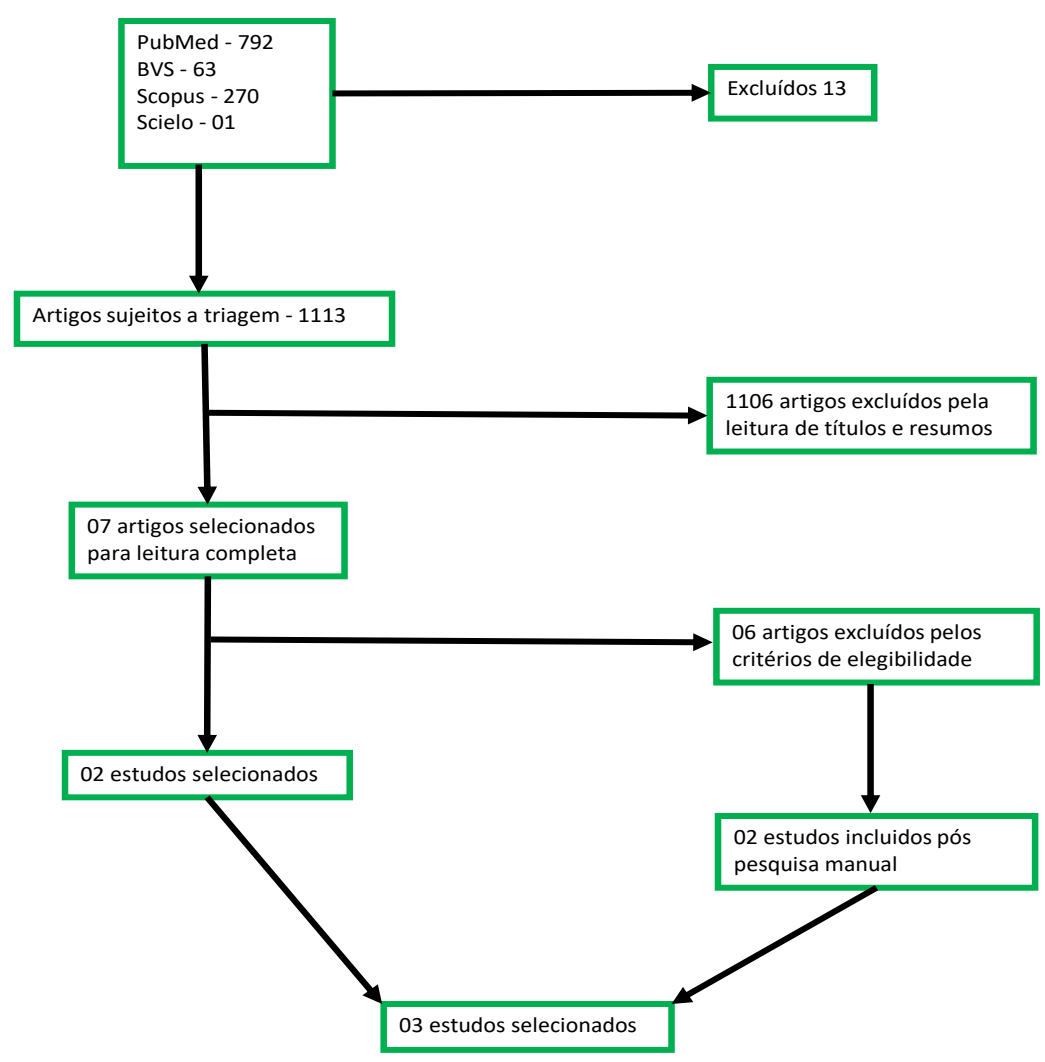

Fonte: Autores.

Dos estudos excluídos pelos critérios de elegibilidade, os de autoria de Warmling (2016), Warmling, Santos, \& Mello (2016), foram retirados da amostra na etapa de leitura completa, por não se tratar de intervenção ou orientações odontológicas, mais de entrevistas com cuidadores sobre prevenção na saúde bucal com pacientes demenciados. No estudo de Weijenberg et 
al. (2013) não houve intervenção de saúde bucal. O artigo objetivou a criação de um protocolo para aumentar a atividade mastigatória. Três estudos de Jablonski (2011a; 2011b; 2018) foram excluídos por serem ensaios clínicos e, por isso, não estão em conformidade com a estratégia PECOS.

Os três artigos admitidos para esta pesquisa foram publicados nos anos de 2010, 2012 e 2019. Dois estudos são coortes Ho et al. (2019), Chen et al. (2010) e um estudo transversal Philips et al. (2012). As características estão descritas no Quadro 2.

Quadro 2- Características dos estudos.

\begin{tabular}{|c|c|c|c|c|c|c|c|c|c|c|}
\hline $\begin{array}{c}\text { Título do } \\
\text { Estudo }\end{array}$ & $\begin{array}{c}\text { Autor/ } \\
\text { ano de } \\
\text { publicação }\end{array}$ & $\begin{array}{l}\text { Desenho } \\
\text { do } \\
\text { estudo }\end{array}$ & População & $\begin{array}{l}\text { Local } \\
\text { da } \\
\text { interven } \\
\text { ção }\end{array}$ & $\begin{array}{l}\text { Tipo de } \\
\text { intervenção } \\
\text { realizada }\end{array}$ & $\begin{array}{l}\text { Profissiona } \\
\text { is } \\
\text { envolvidos } \\
\text { na prática }\end{array}$ & $\begin{array}{c}\text { Tempo } \\
\text { de } \\
\text { investi } \\
\text { gação }\end{array}$ & $\begin{array}{c}\text { Desfech } \\
0\end{array}$ & $\begin{array}{l}\text { Revista } \\
\text { de } \\
\text { publica } \\
\text { ção }\end{array}$ & $\begin{array}{l}\text { Local da } \\
\text { realização do } \\
\text { estudo }\end{array}$ \\
\hline $\begin{array}{l}\text { Effectiveness } \\
\text { of the } \\
\text { implementatio } \\
\text { n project } \\
\text { 'Don't forget } \\
\text { the mouth!' of } \\
\text { community } \\
\text { dwelling older } \\
\text { people with } \\
\text { dementia: a } \\
\text { prospective } \\
\text { longitudinal } \\
\text { single-blind } \\
\text { multicentre } \\
\text { study protocol } \\
\text { (DFTM!) }\end{array}$ & $\begin{array}{l}\text { Ho et al., } \\
2019\end{array}$ & $\begin{array}{l}\text { Estudo de } \\
\text { Coorte } \\
\text { multicêntri } \\
\text { ca simples- } \\
\text { cego, que } \\
\text { aconteceu } \\
\text { em } \\
14 \text { cidades } \\
\text { na } \\
\text { Holanda. }\end{array}$ & $\begin{array}{l}\text { Idosos (65 } \\
\text { anos ou } \\
\text { mais), } \\
\text { inscritos no } \\
\text { atendimento } \\
\text { domiciliar } \\
\text { das cidades }\end{array}$ & $\begin{array}{l}\text { Unidade } \\
\text { de saúde }\end{array}$ & $\begin{array}{l}\text { Grupo } \\
\text { controle: } \\
\text { cuidados } \\
\text { habituais. } \\
\text { Grupo } \\
\text { intervenção: } \\
\text { cuidados } \\
\text { profissionais } \\
\text { (educação em } \\
\text { saúde; avaliação } \\
\text { da higiene oral) }\end{array}$ & $\begin{array}{l}\text { Médicos, } \\
\text { enfermeiros, } \\
\text { clínicos gerais } \\
\text { e distritais, } \\
\text { dentistas, } \\
\text { higienistas } \\
\text { dentais, } \\
\text { gerentes de } \\
\text { caso, } \\
\text { assistentes } \\
\text { sociais e } \\
\text { cuidadores } \\
\text { informais }\end{array}$ & 12 meses & $\begin{array}{l}\text { Melhoria } \\
\text { da higiene } \\
\text { oral com } \\
\text { redução da } \\
\text { placa } \\
\text { dental e da } \\
\text { placa } \\
\text { dentária } \\
\text { protética. }\end{array}$ & $\begin{array}{l}\text { BMC } \\
\text { Oral } \\
\text { Health }\end{array}$ & Holanda \\
\hline $\begin{array}{l}\text { Patterns of } \\
\text { Tooth Loss in } \\
\text { Older Adults } \\
\text { with and } \\
\text { without } \\
\text { Dementia: A } \\
\text { Retrospective } \\
\text { Study Based } \\
\text { on a } \\
\text { Minnesota } \\
\text { Cohort }\end{array}$ & $\begin{array}{l}\text { Chen et al., } \\
2010\end{array}$ & $\begin{array}{l}\text { Estudo de } \\
\text { Coorte } \\
\text { longitudina } \\
1 \\
\text { retrospecti } \\
\text { vo }\end{array}$ & $\begin{array}{l}491 \text { idosos } \\
\text { de St. Paul, } \\
\text { Minnesota }\end{array}$ & $\begin{array}{l}\text { Clínica } \\
\text { odontológ } \\
\text { ica } \\
\text { geriátrica } \\
\text { na ILP. }\end{array}$ & $\begin{array}{l}\text { Tratamento } \\
\text { dentário antes do } \\
\text { início do estudo } \\
\text { (raspagem, } \\
\text { sangramento } \\
\text { gengival e placa } \\
\text { dentária). } \\
\text { Atendimento } \\
\text { odontológico de } \\
\text { rotina } \\
\text { continuamente } \\
\text { (prevenção, } \\
\text { restauração, } \\
\text { periodontia e } \\
\text { cirurgia). } \\
\text { Avaliação de } \\
\text { perda dentária }\end{array}$ & $\begin{array}{l}\begin{array}{l}\text { Profissionais } \\
\text { de } \\
\text { odontologia e } \\
\text { cuidadores }\end{array} \\
\text { Diagnóstico } \\
\text { de demência: } \\
\text { neurologista, } \\
\text { cardiologistas, } \\
\text { psiquiatras, } \\
\text { geriatras e } \\
\text { médicos } \\
\text { clínicos }\end{array}$ & $\begin{array}{l}\text { Sete anos } \\
\text { (outubro } \\
\text { de } 1999 \\
\text { a } \\
\text { dezembr } \\
\text { o de } \\
2006 \text { ) }\end{array}$ & $\begin{array}{l}\text { O padrão } \\
\text { de perda } \\
\text { dentária } \\
\text { não diferiu } \\
\text { entre os } \\
\text { grupos } \\
\text { (com e sem } \\
\text { demência). }\end{array}$ & $\begin{array}{l}\text { Journal of } \\
\text { the } \\
\text { American } \\
\text { Geriatrics } \\
\text { Society }\end{array}$ & $\begin{array}{l}\text { Minnesota - } \\
\text { Estados Unidos }\end{array}$ \\
\hline $\begin{array}{l}\text { Oral hygiene } \\
\text { care status of } \\
\text { elderly with } \\
\text { dementia and } \\
\text { in residential } \\
\text { aged care } \\
\text { facilities }\end{array}$ & $\begin{array}{l}\text { Philip et al., } \\
2012\end{array}$ & $\begin{array}{l}\text { Estudo } \\
\text { transversal }\end{array}$ & $\begin{array}{l}205 \text { idosos } \\
\text { residentes } \\
\text { em Perth, } \\
\text { Austrália } \\
\text { Ocidental }\end{array}$ & $\begin{array}{l}\text { Domicílio } \\
\text { (instalaçõ } \\
\text { es } \\
\text { residencia } \\
\text { is de lar } \\
\text { de idosos) }\end{array}$ & $\begin{array}{l}\text { Exames orais } \\
\text { (índice de placa } \\
\text { bacteriana e de } \\
\text { inflamação } \\
\text { gengival). } \\
\text { Medida das } \\
\text { atividades de } \\
\text { saúde oral da } \\
\text { vida diária } \\
\text { (ADLOH). } \\
\text { Assistência de } \\
\text { higiene oral }\end{array}$ & $\begin{array}{l}\text { Dentista, } \\
\text { Auxiliar de } \\
\text { saúde bucal, } \\
\text { cuidadores }\end{array}$ & $\begin{array}{l}\text { Sete anos } \\
(2002 \mathrm{a} \\
2008)\end{array}$ & $\begin{array}{l}\text { O estado } \\
\text { dos } \\
\text { cuidados } \\
\text { de higiene } \\
\text { bucal em } \\
\text { residentes } \\
\text { com } \\
\text { demência } \\
\text { era ruim, } \\
\text { em relação } \\
\text { aos sem } \\
\text { demência }\end{array}$ & $\begin{array}{l}\text { Gerodont } \\
\text { o-logy }\end{array}$ & $\begin{array}{l}\text { Austrália } \\
\text { Ocidental }\end{array}$ \\
\hline
\end{tabular}

Fonte: Autores.

Os artigos selecionados de Ho et al. (2019), Chen et al. (2010), Philips et al. (2012) relatam a inserção de práticas odontológicas a pacientes idosos com demência.

O estudo de Chen et al. (2010) publicado no Journal of the American Geriatrics Society, teve por objetivo analisar os padrões de perdas dentárias de 491 idosos com demência num lar de idosos em Minnesota, Estados Unidos. Trata-se de um estudo de coorte retrospectivo. Os participantes foram tratados e trazidos ao estado de saúde bucal estável e categorizados em dois grupos, com e sem demência. A frequência das visitas odontológicas foi baseada na percepção das necessidades do 
paciente, observada pelos cuidadores e pelos profissionais de odontologia durante o estudo. Foi constatado que os adultos idosos com demência não têm necessariamente um risco maior de perda de dentes, sendo possível manter a dentição e função oral, assim como os idosos sem demência, se for oferecido a intervenção apropriada.

Com o objetivo de explorar a eficácia de higiene oral em 205 idosos com demência, residentes em ILPs, na Austrália, Philips et al. (2012) investigaram a partir de um estudo transversal a realização de exames orais (índice de placa bacteriana e de inflamação gengival) e medida das atividades diárias de higiene oral (ADHO). Constatou-se, portanto, que o estado de higiene oral e gengival era pobre entre os residentes com demência, em comparação com aqueles sem demência e que os residentes incapacitados e também com demência possuíam escores piores comparados com o resto da amostra.

O estudo mais atual selecionado, foi o de Ho et al. (2019) publicado na revista BMC Oral Health, trata-se de uma coorte, onde foi aplicado o protocolo do projeto "Não esqueça a boca" em 14 cidades da Holanda em pessoas idosas com demência, dispondo de um grupo controle, no qual realizou-se os cuidados habituais e o grupo de intervenção, cuidados profissionais (educação em saúde; avaliação da higiene oral através da presença de placa dentária ou placa dentária protética), durante 12 meses, observado a melhoria da higiene oral com redução da placa dental e da placa dentária protética.

A qualidade metodológica dos estudos incluídos nesta revisão está descrita na Tabela 1. Os escores totais da Escala de Newcastle-Ottawa variaram entre 4 e 7 pontos, sendo um estudo considerado de baixa qualidade, Chen et al. (2010) e dois alcançaram 4 e 3 estrelas para seleção dos grupos Ho et al. (2019) e Philips et al. (2012) respectivamente. Apenas o estudo de Philips et al. (2012) alcançou uma estrela para comparabilidade dos grupos de estudo.

Tabela 1 - Análise da qualidade metodológica dos estudos incluídos

\begin{tabular}{|c|c|c|c|c|c|}
\hline ESTUDO & DESENHO & SELEÇÃO & COMPARABILIDADE & DESFECHO & $\begin{array}{l}\text { TOTAL } \\
\text { SCORE }\end{array}$ \\
\hline $\begin{array}{l}\text { Ho et al. } \\
(2019)\end{array}$ & $\begin{array}{l}\text { Estudo } \\
\text { Coorte }\end{array}$ & 4 & 0 & 3 & $7 / 9$ \\
\hline $\begin{array}{l}\text { Chen et al. } \\
(2010)\end{array}$ & $\begin{array}{l}\text { Estudo } \\
\text { Coorte }\end{array}$ & 2 & 0 & 2 & $4 / 9$ \\
\hline $\begin{array}{l}\text { Philips et } \\
\text { al. } \\
(\mathbf{2 0 1 2})\end{array}$ & $\begin{array}{c}\text { Estudo } \\
\text { Transversal }\end{array}$ & 3 & 1 & 2 & $6 / 7$ \\
\hline
\end{tabular}

Fonte: Autores.

Nos estudos resultantes desta revisão sistemática, o de Ho et al. (2019) alcançou sete estrelas, considerado com risco de viés moderado, enquanto o trabalho publicado por Chen et al. (2010) foi considerado com alto risco de viés. A pesquisa de Philips et al. (2012), o único de desenho transversal, foi avaliado como moderado risco de viés. No estudo de Ho et al. (2019), não houve aleatorização, e o instrumento utilizado não foi validado para uso em pessoas com demência, assim como na pesquisa de Chen et al. (2010) não houve treinamento técnico específico para os examinadores, assim como não foram utilizados critérios objetivos para o diagnóstico da demência por se tratar de um estudo de coorte retrospectivo. 


\section{Discussão}

O objetivo desta revisão sistemática foi analisar as práticas odontológicas em pacientes idosos com demência, considerando que essa população compõe um grupo que requer cuidado especial quanto à sua saúde, incluindo, neste aspecto, a saúde bucal. Durante a investigação, somente três estudos puderam compor a amostra, tendo em vista os critérios de inclusão adotados.

O estudo de Ho et al. (2019) teve como objetivo manter e melhorar a saúde bucal e a saúde geral dos idosos frágeis demenciados, tendo em vista que é relevante a avaliação da repercussão da saúde geral e das situações de vida sobre a saúde bucal, assim como o inverso; ou seja, o efeito da desarmonia da saúde bucal sobre a saúde geral, com consequências dramáticas na qualidade de vida e no bem-estar do idoso (Oliveira et al., 2011).

Ferreira et al. (2014) ao realizar um estudo com o objetivo de identificar a prevalência de idosos com comprometimento cognitivo e identificar associações entre este e as condições de saúde bucal, observou-se que indivíduos com comprometimento cognitivo apresentaram piores condições de saúde bucal do que os idosos com condição cognitiva preservada, incluindo cárie coronária, cárie radicular, raízes retidas, bolsas periodontais, lesões mucosas. Esses achados confirmam os desfechos do estudo de Philips et al. (2012) que mostrou que o estado de higiene oral e gengival era pobre entre os residentes das ILPs com demência, em comparação com aqueles sem demência e que os residentes incapacitados e com demência tinham piores escores em relação ao restante da amostra, no qual valida os resultados apurados pelo estudo de (Zanesco et al., 2018) onde os danos oriundos de condições bucais insatisfatórias, como a dificuldade para alimentar-se em decorrência de problemas nos dentes, edentulismo e nunca ter consultado um dentista, estão significativamente relacionados à probabilidade de dificuldade funcional entre idosos.

Ainda no estudo de Philip et al. (2012) foi identificado como um fator de risco para saúde bucal a assistência de higiene oral e dependência funcional deficiente entre os residentes com demência, além de questionar a capacidade e qualidade dos profissionais em saúde fornecer cuidados orais eficazes onde corroboram com os achados de (Araújo, Andrade \& Pinto, 2020) no qual identificou que pacientes idosos com alguma síndrome demencial apresentam fragilidades que dificultam e/ou impedem a realização de uma higiene bucal de qualidade. O estudo discute a participação dos profissionais da saúde, colaborando com as orientações para os cuidadores e para os próprios pacientes, quando possível, para a realização correta da higiene oral. Os autores também ressaltam que a saúde oral insuficiente desse público se deve a outras causas, como as comorbidades, efeitos colaterais da medicação, disfunção motora e cognitiva; em relação ao cuidador, como falta de experiência ou de priorização dos cuidados de higiene bucal e sobrecarga de tarefas; ou ainda em relação inexistência de políticas públicas para inclusão do cirurgião-dentista no atendimento domiciliar e a ênfase na Odontogeriatria na formação profissional (Rabelo et al., 2020).

Entretanto, o estudo de Chen et al. (2010) diverge desses dados quando relata que os idosos com demência não têm necessariamente um risco maior de perda de dentes, sendo possível manter a dentição e função oral, se oferecida a intervenção adequada. Além disso Santiago, Simões \& Pereira (2008) afirmam que se a saúde bucal pudesse ser focalizada desde o início do diagnóstico de demência, ofereceria outras possibilidades de cuidado, visto que os pacientes com demência são mais capazes física e mentalmente nos estágios iniciais do curso da doença (0-4 anos) quando a maioria dos aspectos da higiene bucal deve ser possível, pois, com a evolução da doença há crescente dificuldade de aceitação e realização do tratamento.

Os pacientes idosos com demência devem ter a sua higiene e saúde bucal melhoradas, podendo ser lançado estratégias de educação em saúde com cuidadores formais e informais, uso de ferramentas de triagem de saúde bucal, bem como atendimento odontológico regular (Delwel et al., 2017). O plano de tratamento deve considerar o estágio demencial, a capacidade cognitiva desse paciente, a presença de comorbidades, uso de medicamentos, bem como estar em consonância com 
a equipe de saúde que assiste o paciente, primando pela sua qualidade de vida e primordialmente eliminando focos de infecções e situações que desencadeiem dor (Miranda \& Montenegro, 2009; Miranda et al., 2010). Corroborando com essas informações, Miranda et al. (2010), consideram que o cirurgião dentista deve ter conhecimento preliminar das várias fases da demência e suas características, de forma a direcionar a atenção à saúde oral individualizada.

Através do estudo de coorte realizado por Ho et al. (2019), identificou-se que o protocolo utilizado na investigação, denominado de "Não esqueça a boca", avaliou a eficácia de implementação de cuidados de saúde bucal para idosos com demência na comunidade, além disso foi um estudo que contou com a colaboração multidisciplinar de profissionais de saúde e cuidadores informais. Para Miranda et al. (2010), a atuação dos cuidadores, familiares, além da equipe de saúde bucal é indispensável e primordial para preservação da saúde oral desses idosos.

Conforme desfechos apontados no estudo de Vaz (2018), o ofício do cirurgião dentista, enquanto prestador de cuidados a idosos com demência, é relevante e desafiador, num atributo de criar uma barreira ou um meio facilitador no tratamento e manutenção da qualidade de vida destes pacientes, tal como a atuação da Odontologia deve estar inserida numa perspectiva integral do paciente, com conhecimentos específicos de diversas áreas, objetivando a promoção e a manutenção da saúde do paciente com demência (Oliveira et al., 2011). Percebe-se então a importância do profissional se aperfeiçoar para esses atendimentos com o propósito de se relacionar de forma inter e multidisciplinar, seja em consultório particular, posto de saúde, domicílio, hospital (centro cirúrgico, Unidade de Terapia Intensiva, enfermaria) (Miranda et al., 2010).

Quanto à qualidade dos estudos incluídos, a avaliação de risco de viés indica serem necessários ajustes ao tentar reproduzir os modelos metodológicos incluídos nesta revisão, com atenção especial à aferição dos dados (Pereira, Galvão \& Freire, 2014).

\section{Conclusão}

Os resultados obtidos nesta revisão sistemática demostraram a necessidade de novos estudos que apresentem a descrição de práticas odontológicas dos idosos com demência, descrevendo práticas de forma reprodutível que gerem evidências de ações preventivas de educação em saúde e de intervenções. Assim, são necessários delineamentos mais rigorosos, com menor risco de viés, como os ensaios clínicos controlados e randomizados, com o objetivo de testar hipóteses e produzir protocolos eficientes para o desenvolvimento de técnicas para as intervenções odontológicas aos idosos com demência. Tendo em vista que este estudo se limitou a analisar estudos observacionais e diante dos resultados obtidos, não foi possível realizar análise estatísticas dos dados, pois não haviam dados disponíveis para executar esta etapa.

O crescimento da população idosa e a necessidade de assistência no território corrobora para o investimento público em treinamentos para as equipes de saúde, com ênfase em colaboração multiprofissional, e destacando-se que os profissionais da Odontologia podem contribuir para orientações de familiares e cuidadores, com o objetivo de manter a higiene oral e reduzir a perda dentária, assim como presença de dor e outros agravos ocorridos em consequência da ausência do cuidado. À vista disto, a intervenção odontológica em pacientes idosos com demência é algo fundamental na atenção primária de saúde, tal como preconizado na Política Nacional de Atenção Básica (PNAB).

\section{Referências}

Araújo, A. S., Andrade, M., \& Pinto, G. A. (2020). Higiene e Saúde bucal em idosos na atenção primária: uma revisão sistemática. Revista Eletrônica Acervo Saúde, 2673.

Cardoso, Dietrich, \& Souza. (2021). Envelhecimento da população e desigualdade. Brazilian Journal of Political Economy, 41 (01) ,23-43.

Chen, X., Shuman, S. K., Hodges, J.S. Gatewood, L.C, Xu, J. (2010). Patterns of Tooth Loss in Older Adults with and without Dementia: A Retrospective Study Based on a Minnesota Cohort. Journal of the american Geriatrics Society, 58 (12), 2300-2307. 
Dantas, C. M. H.L.(2013). Capacidade funcional de idosos com doenças crônicas residentes em Istituições de Longa Permanencia. Revista Brasileira de Enfermagem,66 (06), 914-920.

Delwel, S., Binnekcade, T. T., Perez, R. S. G. M., Hertogh, C.M.P.M., Scherder, E. J. A., Iobbezzoo, F., (2017).Oral health and orofacial pain in older people with dementia: a systematic review with focus on dental hard tissues. Clinical oral investigations, 21 (01), 17-32.

Donato H. \& Donato M. (2019) Etapas na condução de uma revisão sistemática, Acta Med Port 227-235.

Duque, A.M. (2019). Determinantes sociais da saúde e envelhecimento: uma abordagem espaciale temporal da realidade do Brasil e de Sergipe. ( Tese doutorada) Universidade Federal de Sergipe, Aracaju-SE.

Ferreira, R. C., Vargas, A.M.D.,Fernandes, N. C. N., Souza, J. G. S., Sá, M. A. B., Oliveira, L.F.B., Martins, A. M. E. B. (2014). O idoso com comprometimento cognitivo apresenta pior condição bucal? Ciencias \& Saúde Coletiva, 19 (08), 3417-3428.

Fereshtehnejad, S.M., Ptacek, S.G., Religa, D.D., Holmer, J., Buhlin, K., Eriksdotter, M., Englund, S.G. (2018). Dental care utilization in patients with different types of dementia: A longitudinal nationwide study of 58, 037 individuals. Alzheimer's \& Dementia, 14 (01), 10-19.

Galvão, Taís Freire, \& Pereira, Mauricio Gomes. (2014). Revisões sistemáticas da literatura: passos para sua elaboração. Epidemiologia e Serviços de Saúde, 23(1), 183-184

Hatipoglu, M.G., Kabay, S.C., Guven, G. (2011) The clinical evaluation of the oral status in Alzheimer-type dementia patients. Gerodontology.28 (04), 302306

Ho, B.V., Weijenberg, R.A.F., Maarel-Wierink, C.D.V., Visscher, C.M., Putten, G.V., Scherder, E.J.A.S., Lobbezoo, F. (2019). Effectiveness of the implementation project " Dont't forget the mouth' of community dwelling older people with dementia: a prospective longitudinal single-blind multicentre stydy protocol (DFTM!). BMC Oral Heath, $19(01), 91$.

Instituto Brasileiro de Geografia e Estatística. (2013). Projeção da população brasileira por sexo e idade: 2000 -2060.

Jablonski, R. A., Therrien, B., Mahoney, E. K., Kolanowski, A., Gabello, M., Brock, A. (2011a). An intervention to reduce care-resistant behavior in persons with dementia during oral hygiene: a pilot study. Special Care Dentistry, 31 (03),77-87.

Jabloski, R. A., Kolanowski,A.M., Therrien, B., Mahoney, E.K., Kassab, C., Leslie, D.L. (2011b). Reducing care-resistant behaviors during oral hygiene in persons with dementia. BMC Oral Health, 11 (30),1-10.

Jablonski, R. A., Kolanowski,A.M.,Azuero, A., Winstead, V., Jones-Townsend, C., Geisinge,r M. L. (2018). Randomised clinical trial: Efficacy of strategies to provide oral hygiene activities to nursing home residents with dementia who resist mouth care. Gerondontology, 35 (04),1-11.

Miranda, A. F., Miranda, M. P.A. F., Lia, E. N.,Leal, S. C. (2010). Doença de Alzheimer : caracter’sticas e orientações em Odontologia. RGO- Revista Gaúcha de Odontologia,58 (01), 103-107.

Miranda, A. F., \& Montenegro, F. L. (2009). Atuação Odontológica na doença de Alzheimer: relato de caso clinico multidisciplinar. International Journal of Dentistry, 08 (04), 220-224.

Oliveira, R. M.T., Lia, E. N., Macedo, S. B., Amorim, R. F. B.(2011). Status da Saúde Bucal em Pacientes com Demência Senil. Revista Odontológica do Brasil Central, 20 (53), 114-118.

Organização Mundial de Saúde. (2005). Envelhecimento ativo: uma politica de saúde. Brasilia DF: Organização Pan Americana de saúde.

Paim, J., Travassos, C., Almeida, C., Bahia, L., Macinko, J. (2011). Sistema de saúde brasileiro: história, avanços e desafios. Lancet, 03,11-31.

Patra, J., Bhatia, M., Suraweera, W., Morris, S.K., Patra, C., Gupta, P.C., Jha, P. (2015). Exposure to Second-Hand Smoke and the Risk of Tuberculosis in Children and Adults: A Systematic Review and Meta- Analysis of 18 Observational Studies. Plos Medicine,12 (06), 1-21.

Pereira, Galvão, M. G., \& Freire, T. (2014). Heterogeneidade e viés de publicação em revisões sistemáticas. Epidemiologia e serviços de saúde [on line], 23 (04),775-778

Philips, P. ,Roger, C., Kruger E., Tennant, N. (2012). Oral hygiene care status of elderly with dementia and in residential aged care facilities. Gerondontology, 29,306-311.

Rabelo, R. G., Mello S. M. F., Neto, A. T., Araújo, N. S. (2020). A doença de Alzheimer e o cuidado em saúde bucal. Revista da Faculdade de Odontologia da uNiversidade Federal da Bahia, 50 (01),71-77.

Santiago, E., Simões, R. J., \& Pereira, J. A. (2008). A Saúde Oral na Doença de Alzheimer. Arquivo de Medicina, 22 (06),189-193.

Terwee, C. B., Bot, S.D.M., Boer, M.R., Windt, D.A., Knol, D.L., Dekker, J., Bouter, L.M., Vet, H.C. (2007). Quality critera were proposed for measurement properties of heath status questionnaires. Journal of Clinical Edpidemiology, 60 (01),34-42

Vaz, J. R. (2018). Saúde Oral em Pacientes com Demência. (Tese Mestrado) Instituto Universitário Egas Moniz-Almada, Portugal.

Warmling, A. M. (2016). Cuidado à Saúde bucal de idosos com doença de Alzheimer no âmbito domiciliar. (Tese de Doutorado) Universidade Federal de Santa Catarina. Santa Catarina-SC.

Warmling, A. M., Santos, S. M., \& Mello, A. L. (2016). Estratégias de cuidado bucal para idosos com doença de Alzheimer no domicilio. Revista Brasileira de Geriatria e Gerontologia, 19 (05),851-860. 
Research, Society and Development, v. 10, n. 9, e59510918367, 2021

(CC BY 4.0) | ISSN 2525-3409 | DOI: http://dx.doi.org/10.33448/rsd-v10i9.18367

Weijenberg, R. A. F., Lobbezoo, F., Knol, D. L., Tomassen, J., Scherder, E. J. A. (2013). Increased masticatory activity and quality of life in elderly persons with dementia- a longitudinal matched cluster randomized single-blind multicenter intervention study. BMC Neurology, 13,26.

Wells, B. S. (1999). The Newcastle-Ottawa Scale (NOS) for Assessing the Quality of Nonrandomised Studies in Meta-Analyses. Ottawa Hospital Research Institute.

Zanesco, C., Bordin, D., Santos, C. B., Fadel, C. B.(2018). Implicações da Saúde bucal na esfera funcional de idosos brasileiros. Revista Odontológica da UNESP,47 (05), 267-281.

Zenthöfer, A., Schröder, J., Cabrera, T., Rammelsberg, P., Hassel AJ. (2014) Comparason of oral health among older people with and without dementia. Community Dental Heath Journal,31 (01), 27-30. 